\title{
Infants Experience Perceptual Narrowing for Nonprimate Faces
}

\author{
Elizabeth A. Simpson, Krisztina Varga, Janet E. Frick, \\ and Dorothy Fragaszy \\ Psychology Department \\ University of Georgia
}

\begin{abstract}
Perceptual narrowing - a phenomenon in which perception is broad from birth, but narrows as a function of experience - has previously been tested with primate faces. In the first 6 months of life, infants can discriminate among individual human and monkey faces. Though the ability to discriminate monkey faces is lost after about 9 months, infants retain human face discrimination, presumably because of their experience with human faces. The current study demonstrates that 4- to 6-month-old infants are able to discriminate nonprimate faces as well. In a visual paired comparison test, 4- to 6-month-old infants $(n=26)$ looked significantly longer at novel sheep (Ovis aries) faces, compared to a familiar sheep face $(p=.017)$, while 9- to 11-month-olds $(n=26)$ showed no visual preference, and adults $(n=27)$ had a familiarity preference $(p<.001)$. Infants' face recognition systems are broadly tuned at birth — not just for primate faces, but for nonprimate faces as well-allowing infants to become specialists in recognizing the types of faces encountered in their first year of life.
\end{abstract}

Much debate has surrounded the special nature of face processing. Interestingly, our sensitivity to faces is not limited to human faces, but extends to

Correspondence should be sent to Elizabeth Simpson, Psychology Department, University of Georgia, 125 Baldwin Street, Athens, GA 30601. E-mail: simpsone@uga.edu Krisztina Varga is now at the Department of Psychology, James Madison University. Results from this experiment were presented at the Cognitive Development Society Conference, San Antonio, TX. Part of this report will be submitted by the first author in partial fulfillment of the Ph.D. degree in Psychology at the University of Georgia. 
the faces of other animals. Research suggests that young infants (e.g., younger than 6 months old) may be better than adults at discriminating faces of nonhuman animals. Pascalis, de Haan, and Nelson (2002) found 6-montholds, 9-month-olds, and adults could discriminate human faces, but only 6month-olds could discriminate monkey faces (Pascalis et al., 2002). The authors concluded that this species-specific effect might reflect perceptual narrowing, a phenomenon that occurs for faces, much like that for speech sounds. For example, between 6- and 12-months of age, discrimination of speech sounds not in one's native language declines (Werker \& Tees, 2005). Perceptual narrowing is dependent on experience; infants selectively discriminate among native phonemes, whereas discrimination of phonemes to which they are not exposed declines. Perceptual narrowing has been characterized as a fine-tuning of perception with age (Nelson, 2001). From birth, perception starts out broadly tuned to all stimuli, both relevant and irrelevant, but narrows during the first year of life (by about 9- to 12-months of age), processing relevant stimuli in greater depth. Perceptual narrowing is a widespread developmental phenomenon, that occurs for vocalizations (Vouloumanos, Hauser, Werker, \& Martin, 2010), face-voice matching (Lewkowicz \& Ghazanfar, 2006; Lewkowicz, Leo, \& Simion, 2010; Pons, Lewkowicz, Sebastián-Gallés, \& Soto-Faraco, 2009; see also Zangenehpour, Ghazanfar, Lewkowicz, \& Zatorre, 2009), musical structure (Hannon \& Trehub, 2005; Trehub \& Hannon, 2006), and visual language processing (Weikum et al., 2007).

As suggested by Pascalis et al. (2002), perceptual narrowing also occurs for facial identity discrimination (i.e., recognizing whether a face is new or familiar). According to this view, rather than being born human face specialists, infants are born face generalists, and become human face experts with experience. Perceptual narrowing of facial identity occurs for face ethnicity (e.g., Kelly et al., 2007), gender (e.g., Quinn, Yahr, Kuhn, Slater, \& Pascalis, 2002), and age (e.g., Cassia, Kuefner, Picozzi, \& Vescovo, 2009).

It is unknown whether human infants' perceptual narrowing for facial identity occurs for nonprimates, as researchers have only examined phylogenetically closely related species (Old World monkeys and humans: Pascalis et al., 2002, 2005; Scott \& Monesson, 2009) and companion animals (dogs and cats: Quinn \& Eimas, 1996). Quinn and Eimas (1996) found that 3- to 4month-old infants are able to discriminate facial identity for both cat and dog faces; however, these data cannot be used to determine whether perceptual narrowing occurs for nonprimate faces as Quinn and Eimas did not examine whether this ability declines in later infancy (e.g., after 9 months). In addition, some infants have experience with cats and dogs as pets, so this may have influenced the 3- to 4-month-olds' discrimination abilities in the Quinn and Eimas study. More recently, 6- to 24-month-old infants matched 
emotional dog facial expressions and vocalizations (Flom, Whipple, \& Hyde, 2009); however, perceptual narrowing may not have occurred because many infants have experiences with dogs. In addition, faces and voices were matched on the basis of emotional information, not facial identity. Therefore, it remains unknown whether human infants can discriminate facial identity alone for more distantly related species.

The current study examined whether human infants' perceptual narrowing of facial identity occurs for an unfamiliar and phylogenetically more distantly related species: sheep (Ovis aries). It could be that human infants are born with the ability to discriminate all or some primate faces, but not nonprimate animal faces. To this end, we tested face recognition in 4- to 6-month-olds, 9- to 11-month-olds, and adults, who viewed sheep faces in a passive viewing visual paired comparison (VPC) task (Fagan, 1970). Sheep were chosen because of their phylogenetic distance from humans, being more distantly related than species studied previously. In addition, most infants and adults have very little exposure to sheep faces, relative to human faces or common pets, such as dogs. Moreover, adult sheep vary in their facial characteristics, and sheep can recognize one another using such characteristics (Kendrick, da Costa, Leigh, Hinton, \& Peirce, 2001). The present study allowed for a test of whether humans can also recognize sheep based on these facial characteristics.

\section{Purpose and predictions}

The purpose of the present experiment was to examine differences in how 4- to 6-month-olds, 9- to 11-month-olds, and adults discriminate sheep facial identity in a passive-viewing VPC task. Testing all three age groups in the same manner allowed for direct comparisons among groups. We predicted that 4- to 6-month-olds-who have not yet undergone perceptual narrowing for facial identity - would be able to discriminate sheep faces, as reflected by a novelty preference. In contrast, we predicted that 9- to 11-month-olds and adults would not be able to discriminate sheep faces, as reflected by no visual preferences.

\section{METHOD}

\section{Participants}

Twenty-six 4- to 6-month-olds (age range $=129-188$ days; 17 females), and twenty-six 9- to 11-month-olds (age range $=274-458$ days; 14 females), were included in the final data set. All infants were healthy and full-term. Names were found using newspaper birth notices, and families were 
contacted by telephone. Twenty-seven adults (age range $=19-33$ years; 13 females) were recruited through the research participant pool of a large southern university. These sample sizes do not include five 4- to 6-montholds, who were excluded because of fussiness $(n=2)$ or equipment malfunction $(n=3)$, and one 9- to 11-month-old, who was excluded because of fussiness.

\section{Apparatus}

Testing took place within a darkened and quiet room. A $2.3 \times 1.3 \mathrm{~m}$ black curtain enclosed the area on three sides to prevent distraction. Infants sat in an infant seat or on their parent's lap. All participants were seated $60 \mathrm{~cm}$ from the presentation screen $(43 \times 58 \mathrm{~cm})$. The stimuli were presented using rear projection on an InFocus projector (model LT755; Portland, OR). Each session was recorded using two Panasonic VHS cameras (model AG-188Proline; Secaucus, NJ). One camera recorded the participant and the other recorded the stimulus presented on the screen. These images were combined using a Videonics Digital Video Mixer (model MX-1; Campbell, CA). Behaviors were coded offline using the Noldus Observer 5.0; Asheville, NC. The stimuli were presented using Inquisit software by Millisecond (http:// www.millisecond.com), version 2.0.61004.7.

\section{Stimuli}

Thirty sheep photos were provided by Keith Kendrick et al., 2001 of Cambridge University and were confirmed by Reefmann, Kaszàs, Wechsler, \& Gygax (2009) to be neutral expressions. All photos were of different individuals. AdobePhotoshop ${ }^{\mathrm{TM}}$ software (San Jose, CA) was used to center, crop, and adjust the size of all photos to $400 \times 400$ pixels $\left(10^{\circ} \times 16^{\circ}\right.$ on the presentation screen), as well as to adjust the contrast and brightness. Necks, shoulders, and ear tags were removed. All photos were given uniform white backgrounds (see Figure 1).

Prior to each trial, all participants viewed a dynamic centering stimulus (e.g., flashing bull's-eye or cartoon character) that was $12^{\circ} \times 12^{\circ}$ on the presentation screen, and lasted 1,500 msec.

\section{Procedure}

Each participant was randomly assigned to one of two conditions, each with a different familiar face. First, participants were familiarized with a face through the Continuous Familiarization Technique (Rose, Feldman, \& Jankowski, 2002) in which two identical faces were shown, side by side, until 


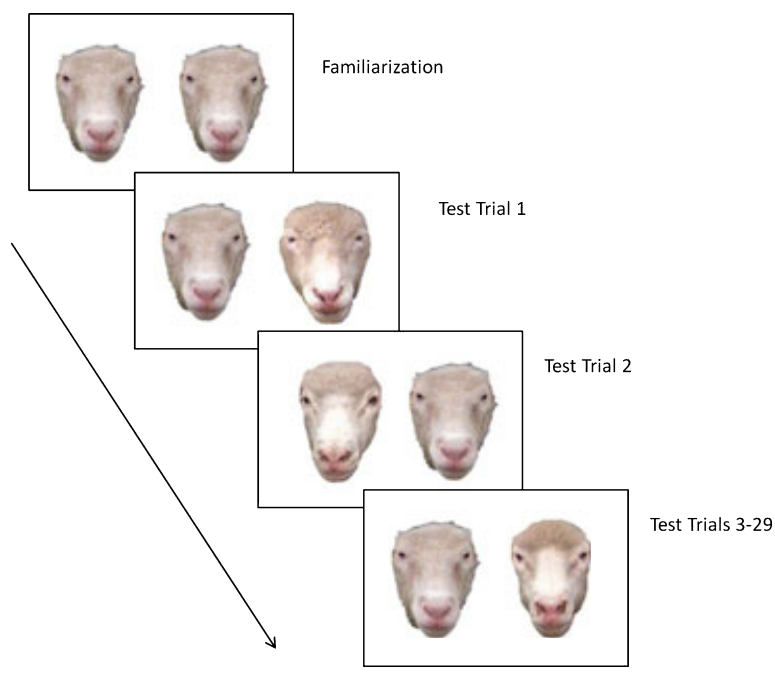

Figure 1 Sample presentation sequence. Participants were first familiarized with a face (familiarization), then viewed a novel and familiar face on 29 subsequent test trials (paired comparison).

the participant accumulated $20 \mathrm{sec}$ of looking to one or both faces (Figure 1). Next, participants viewed 29 paired comparisons, consisting of pairs of faces: One face was the same as that shown in the familiarization phase (i.e., familiar face) and one face was novel. In keeping with other researchers (e.g., Rose et al., 2002), we chose to use 29 test trials so we could examine both the emergence, and the durability, of looking preferences that have been shown to change over the course of numerous test trials in adults (Park, Schimoho, \& Shimojo, 2010) and infants (Rose et al., 2002). The 29 novel faces were presented in a random order, and appeared on the right and left with equal probability. Faces remained on the screen until a cumulative looking time of $4 \mathrm{sec}$ was established; between each trial there was a $1,500 \mathrm{msec}$ intertrial interval that included the centering stimulus. Participants' gaze behaviors - durations of looks toward and away from the novel and familiar faces - were coded off-line for analysis. Testing continued until all 29 trials were completed, or until the participant (or parent) wished to end the experiment.

\section{Coding training and reliability}

All participants' eye movements were coded off-line frame-by-frame (resolution of measurement is $33 \mathrm{msec}$ ) from video using the Noldus Observer 5.0. Observers recorded the location (i.e., left face, right face, away) and 
duration (i.e., start and stop times) of participants' looks during the familiarization phase and paired comparison test trials. Observers were blind to the location of the familiar and novel faces. Interobserver reliability was assessed for the frequency of the look location for each observer. The average level of agreement among observers was 92\% (range 81-97\%).

\section{RESULTS}

A preliminary analysis revealed no gender differences, no effect of the side of the novel face, and no differences across the two familiar faces, so data were collapsed across gender, side of novel face, and familiarization stimulus in subsequent analyses. A one-way analysis of variance (ANOVA) revealed no differences in the total looking time during the familiarization among the three age groups: 4- to 6-month-olds $(M=22.8 \mathrm{sec}, S D=9.0), 9$ - to 11 month-olds $(M=21.0 \mathrm{sec}, S D=4.6)$, and adults $(M=20.7 \mathrm{sec}, S D=$ $1.5), F(2,73)=.90, p=.41$.

To examine whether participants discriminated the novel and familiar sheep faces, we examined the total amount of time looking to each face type, across the first four test trials. Four test trials were chosen because all of the infants in the sample completed at least this many test trials. In addition, novelty preferences have been found in previous work with as few as two test trials (Pascalis et al., 2002). We conducted a $3 \times 2$ mixed design ANOVA that examined the within-subjects factor of face type (novel, familiar) and the between-subjects factor of age group (4- to 6-month-olds, 9- to 11month-olds, adults). We found an interaction between age and face type, $F(2,76)=15.32, p<.001, \eta^{2}=.29$. Paired samples $t$ tests were then conducted for each age group to determine the general patterns of looking during these first four test trials. As depicted in Figure 2, 4- to 6-month-olds show a novelty preference, $t(25)=2.57, p=.017, d=.50$, preferring to look at novel faces $(M=2.75, S D=1.14)$ over familiar faces $(M=2.13$, $S D=.64)$. In contrast, 9- to 11-month-olds demonstrated no looking preferences, $t(25)=.16, p=.87$, looking equally long to the novel faces $(M=2.40, S D=.78)$ and familiar faces $(M=2.36, S D=.61)$. Adults showed a familiarity preference, whereby they looked longer at the familiar $(M=2.65, S D=.54)$ compared to the novel $(M=1.67, S D=.54)$ faces, $t(26)=5.68, p<.001$.

Finally, we examined whether there were overall looking preferences for all trials each participant completed (at least four, but up to 29 trials). A one-way ANOVA revealed that age influenced the proportion of time looking to the novel faces for the entire set of 29 test trials, $F(2,77)=$ $10.58, p<.001, \eta^{2}=.01$; however, follow-up paired comparison $t$ tests 


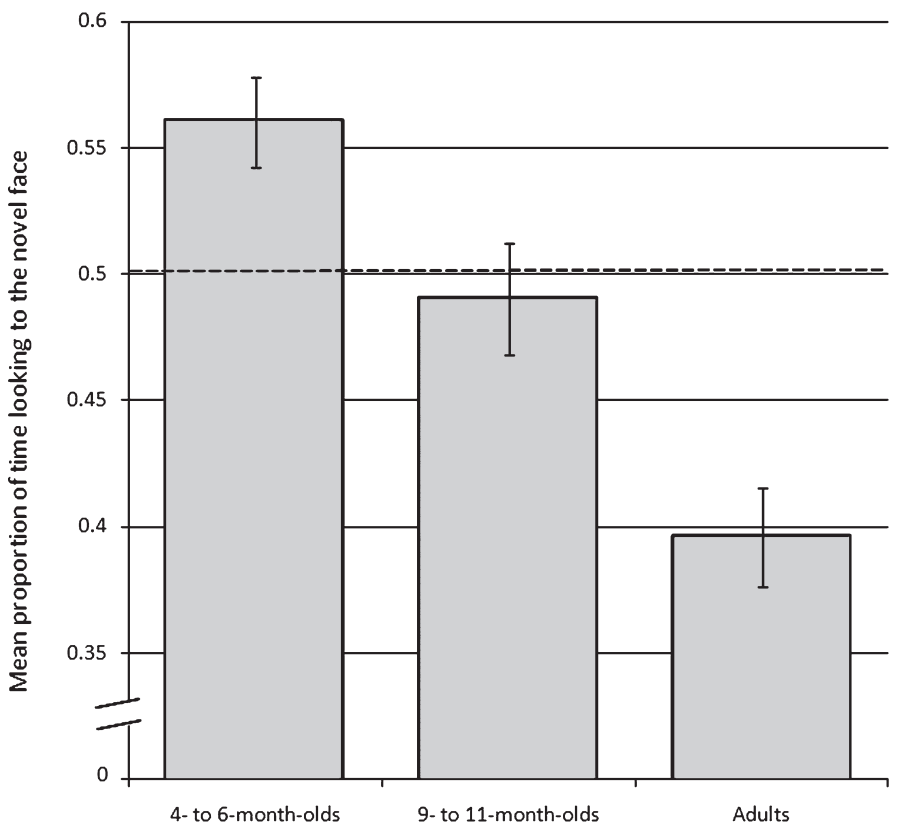

Figure 2 Mean proportion of time looking to the novel face, out of the total amount of time looking to the faces, for the first four trials. Error bars represent standard error of the mean. Dashed line indicates chance performance $(50 \%)$.

examining the mean look durations to novel and familiar faces indicated that only adults showed a preference that lasted throughout the entire 29 trials, consistently preferring the familiar face, $t(26)=4.42, p<.001$, $d=.85$. Neither infant group revealed any overall differences in the proportion of time looking to the novel faces $(p s>.50)$ when all trials were included, suggesting that infants' discrimination skills on the first four trials may have faded as more time elapsed since familiarization.

\section{DISCUSSION}

The aim of the current study was to investigate whether infants and adults could discriminate individual faces of a nonprimate species. The results reported here provide evidence that young infants are able to discriminate unfamiliar nonprimate faces, but that this ability declines in the first year, then appears as a familiarity preference in adulthood. Below, we will interpret these data within a perceptual narrowing framework. 
First, we found that 4- to 6-month-olds demonstrate a novelty preference for sheep faces, which is in line with our predictions, and indicates that young infants have not yet undergone perceptual narrowing. In addition, this finding provides evidence that face discrimination abilities in early infancy (before about 6 months) are even broader than previously thought. That is, not only can young infants discriminate primate faces, and the faces of familiar animals (dogs and cats), but they also have the ability to discriminate the faces of unfamiliar phylogenetically more distantly related (nonprimate) species.

Also consistent with our predictions is the finding that 9- to 11-montholds did not discriminate sheep faces. This is consistent with the developmental pattern of perceptual narrowing, and indicates that older infants have already undergone perceptual narrowing. It is important to keep in mind, however, that older infants' failure to discriminate in the present investigation may be a consequence of their need for a longer familiarization time in order to discriminate. For example, 12-month-olds are able to discriminate monkey faces in a VPC task when familiarized for $40 \mathrm{sec}$, then tested in trials lasting $10 \mathrm{sec}$ (Flom, 2010). Thus, 9- to 11-month-olds' failure to discriminate may not reflect an inability to discriminate, but rather the fact that discrimination is more challenging at this age. Nonetheless, the fact that discrimination of nonprimate faces is either lacking or especially difficult at this age is still consistent with perceptual narrowing.

Though we predicted adults would show no evidence of discriminating the sheep faces - consistent with Pascalis et al.'s (2002) findings that adults cannot discriminate monkey faces in the VPC task-adults showed a familiarity preference. Such a result is consistent with the idea that adults - who have undergone perceptual narrowing - have more difficulty recognizing animal faces. These results fit with the model presented by Hunter (1988) regarding exploratory behavior, which he proposed follows a consistent pattern throughout development. Specifically, Hunter argued that participants show familiarity preferences for stimuli that are more difficult to discriminate, for which they need longer familiarization times to develop a novelty preference. That is, if the stimulus is not well encoded, participants will exhibit a familiarity preference. As familiarization time increases, preferences shift from the familiar to the novel (for a review see Pascalis \& de Haan, 2003). In contrast, participants show a novelty preference for more easily discriminated stimuli, for which they need a shorter familiarization time. Hunter's model would lead to the interpretation that adults in the present study were not familiarized for long enough. Though both novelty preferences and familiarity preferences allow us to infer discrimination, these theoretical models argue that the underlying processes behind the two types of discrimination are not the same. Event-related potential work suggests that 
there are different neural correlates of novelty and familiarity preferences (for a review see Pascalis \& de Haan, 2003). Moreover, while novelty preferences are generally accepted as indicative of declarative or explicit memory, familiarity preferences may indicate residual nondeclarative or implicit memory (Richmond, Colombo, \& Hayne, 2007).

Finally, we examined all completed test trials (up to 29) to determine whether looking behavior patterns persisted over time. When all trials were averaged, no preferences for the novel sheep face were observed in either infant group. Thus, the novelty preference demonstrated by the younger infants appears to be somewhat transient; however, this may be because of inattention and disinterest in the photographs over the course of numerous trials, or a fading of the memory of the familiarization stimulus as more time elapsed since familiarization. Adults, in contrast, demonstrated a lasting familiarity preference, that persisted over the course of all 29 test trials. Infants' perceptual narrowing responses seem to be apparent most strongly in the initial response to these stimuli; adults, in contrast, maintained focused attention for a greater number of trials.

Two possible explanations exist for the differential discrimination of different species that has been found in the present investigation, as well as in previous work. One possibility is that there are differences in the way that human and animal faces are recognized; this is the most common interpretation of the data (e.g., Pascalis et al., 2002; Scott \& Monesson, 2009; Scott, Pascalis, \& Nelson, 2007; Sugita, 2008). A single prototype primate face may subserve face recognition for all primate faces, but this system is not employed for nonprimate faces (Campbell, Pascalis, Coleman, Wallace, \& Benson, 1997).

Another possibility is that differential discrimination across species is because of different amounts of interindividual variability within each species. Variability in facial features is not equivalent across species (Simpson, Varga, Frick, \& Fragaszy, 2010); therefore, better discrimination for any given species may simply be because of the fact that those faces are more heterogeneous. In other words, apparent differences in face discrimination across species may be because of uncontrolled interstimulus perceptual (physical) variance. Consequently, comparisons among conditions that differ concurrently in species and in interstimulus perceptual variance are difficult to interpret (Knebel, Toepel, Judry, le Coutre, \& Murray, 2008).

Additional work must test which of these possibilities best accounts for previous findings. By systematically varying aspects of human and animal faces - such as the feature spacing or head contours - we can determine if equivalent variations in faces across species are distinguished at equal rates across development. Such a test would also allow an examination of what 
strategies individuals of different ages use to discriminate faces, and whether these strategies vary as a function of the species viewed.

\section{ACKNOWLEDGMENT}

This research was supported in part by the J. William Fanning Fellowship and Dissertation Completion Award from the University of Georgia Graduate School.

\section{REFERENCES}

Campbell, R., Pascalis, O., Coleman, M., Wallace, S. B., \& Benson, P. J. (1997). Are faces of different species perceived categorically by human observers? Proceedings of the Royal Society of London B, 264, 1429-1434.

Cassia, V. M., Kuefner, D., Picozzi, M., \& Vescovo, E. (2009). Early experience predicts later plasticity for face processing. Psychological Science, 20, 853-859.

Fagan, J. F. (1970). Memory in the infant. Journal of Experimental Child Psychology, 9, $217-$ 226.

Flom, R. (2010, March). Twelve-month-olds' discrimination of monkey faces: Evidence for perceptual narrowing? Poster session presented at the International Conference on Infant Studies, Baltimore, MD. Abstract retrieved from http://icis2010.isisweb.org/schedule/ dayview.cfm? code $=3$

Flom, R., Whipple, H., \& Hyde, D. (2009). Infants' intermodal perception of canine (Canis familairis) facial expressions and vocalizations. Developmental Psychology, 45, 1143-1151.

Hannon, E. E., \& Trehub, S. E. (2005). Tuning in to musical rhythms: Infants learn more readily than adults. Proceedings of the National Academy of Sciences, 102, 12639-12643.

Hunter, M. A. (1988). A multifactor model of infant preferences for novel and familiar stimuli. Advances in Infancy Research, 5, 69-95.

Kelly, D. J., Quinn, P. C., Slater, A. M., Lee, K., Ge, L., \& Pascalis, O. (2007). The other-race effect develops during infancy: Evidence of perceptual narrowing. Psychological Science, 18, 1084-1089.

Kendrick, K. M., da Costa, A. P., Leigh, A. E., Hinton, M. R., \& Peirce, J. W. (2001). Sheep don't forget a face. Nature, 414, 165-166.

Knebel, J., Toepel, U., Judry, J., le Coutre, J., \& Murray, M. M. (2008). Generating controlled image sets in cognitive neuroscience research. Brain Topography, 20, 284-289.

Lewkowicz, D. J., \& Ghazanfar, A. A. (2006). The decline of cross-species intersensory perception in human infants. Proceedings of the National Academy of Sciences, 103, 6771-6774.

Lewkowicz, D. J., Leo, I., \& Simion, F. (2010). Intersensory perception at birth: Newborns match nonhuman primate faces and voices. Infancy, 15, 46-50.

Nelson, C. A. (2001). The development and neural basis of face recognition. Infant and Child Development, 10, 3-18.

Park, J., Schimoho, E., \& Shimojo, S. (2010). Roles of familiarity and novelty in visual preference judgments are segregated across object categories. Proceedings of the National Academy of Sciences, Early Edition. http://www.pnas.org/cgi/doi/10.1073/pnas. 1004374107 
Pascalis, O., \& de Haan, M. (2003). Recognition memory and novelty preference: What model? In H. Hayne \& J. Fagen (Series Eds.), Progress in infancy research. (Vol. 3, pp. 95-120). Mahwah, NJ: Lawrence Erlbaum Associates.

Pascalis, O., de Haan, M., \& Nelson, C. A. (2002). Is face processing species-specific during the first year of life? Science, 296, 1321-1323.

Pascalis, O., Scott, L. S., Kelly, D. J., Shannon, R. W., Nicholson, E., Coleman, M., \& Nelson, C. A. (2005). Plasticity of face processing in infancy? Proceedings of the National Academy of Sciences, 102, 5297-5300.

Pons, F., Lewkowicz, D. J., Sebastián-Gallés, S., \& Soto-Faraco, N. (2009). Narrowing of intersensory speech perception in infancy. Proceedings of the National Academy of Science, 106, 10598-10602.

Quinn, P. C., \& Eimas, P. D. (1996). Perceptual cues that permit categorical differentiation of animal species by infants. Journal of Experimental Child Psychology, 63, 189-211.

Quinn, P. C., Yahr, J., Kuhn, A., Slater, A. M., \& Pascalis, O. (2002). Representation of the gender of human faces by infants: A preference for female. Perception, 31, 1109-1121.

Reefmann, N., Kaszàs, F. B., Wechsler, B., \& Gygax, L. (2009). Ear and tail postures as indicators of emotional valence in sheep. Applied Animal Behaviour Science, 118, 199-207.

Richmond, J., Colombo, M., \& Hayne, H. (2007). Interpreting visual preferences in the visual paired-comparison task. Journal of Experimental Psychology: Learning, Memory, and Cognition, 33, 823-831.

Rose, S. A., Feldman, J. F., \& Jankowski, J. J. (2002). Processing speed in the 1st year of life: A longitudinal study of preterm and full-term infants. Developmental Psychology, 38, 895-902.

Scott, L. S., \& Monesson, A. (2009). The origin of biases in face perception. Psychological Science, 20, 676-680.

Scott, L. S., Pascalis, O., \& Nelson, C. A. (2007). A domain-general theory of the development of perceptual discrimination. Current Directions in Psychological Science, 16, 197-312.

Simpson, E., Varga, K., Frick, J., \& Fragaszy, D. (2010). Face recognition strategies that underlie infant and adult face discrimination differ for human and animal faces. Manuscript in preparation.

Sugita, Y. (2008). Face perception in monkeys reared with no exposure to faces. Proceedings of the National Academy of Sciences, 105, 394-398.

Trehub, S. E., \& Hannon, E. E. (2006). Infant music perception: Domain-general or domainspecific mechanisms? Cognition, 100, 73-79.

Vouloumanos, A., Hauser, M. D., Werker, J. F., \& Martin, A. (2010). The tuning of human neonates' preference for speech. Child Development, 81, 517-527.

Weikum, W. M., Vouloumanos, A., Navarra, J., Soto-Faraco, S., Sebastián-Gallés, N., \& Werker, J. F. (2007). Visual language discrimination in infancy. Science, 316, 1159.

Werker, J. F., \& Tees, R. C. (2005). Speech perception as a window for understanding plasticity and commitment in language systems of the brain. Developmental Psychobiology, 46, 233251.

Zangenehpour, S., Ghazanfar, A. A., Lewkowicz, D. J., \& Zatorre, R. J. (2009). Heterochrony and cross-species intersensory matching by infant vervet monkeys. PLOS ONE, 4, e4302. 